\title{
TEMPlateTAGger V1.0.0 \\ A Template Matching Tool for Jet Substructure
}

\author{
Mihailo Backović, José Juknevich \\ Department of Particle Physics and Astrophysics \\ Weizmann Institute of Science, Rehovot 76100, Israel
}

\begin{abstract}
TemplateTagger is a $\mathrm{C}++$ package for jet substructure analysis with Template Overlap Method. The code operates with arbitrary models within fixed-order perturbation theory and arbitrary kinematics. Specialized template generation classes allow the user to implement any model for a decay of a boosted heavy object. In addition to Template Overlap, the code provides ability to calculate other template shape and energy flow observables. We describe in detail the structure of the package, as well as provide examples of its usage.
\end{abstract}




\section{Contents}

1 Introduction 3

2 Physics Overview 4

3 Template Overlap in a Pileup Environment 6

3.1 Effects of Pileup on Peak Overlap . . . . . . . . . . . . . . . 6

3.2 Using Multiple Template $p_{T}$ bins in a High Pileup Environment . . . . . . . . . . 6 6

4 Program Structure and Use 8

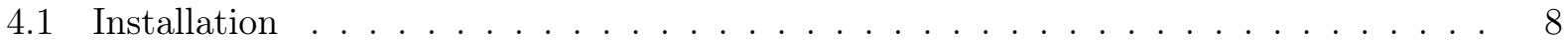

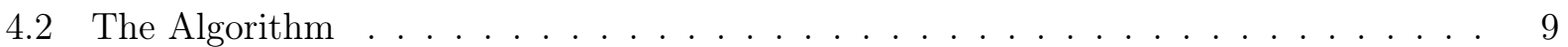

4.3 Efficient Generation of Template Libraries . . . . . . . . . . . . . . . . . . . 10

4.4 Choosing the Kernel . . . . . . . . . . . . . . . . . . . . 13

4.5 Finding the Best Matched Templates . . . . . . . . . . . . . . . . . . . 14

4.6 Sample Program . . . . . . . . . . . . . . . . . . . 17

5 Miscellaneous Tools $\quad 19$

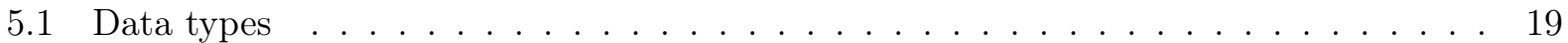

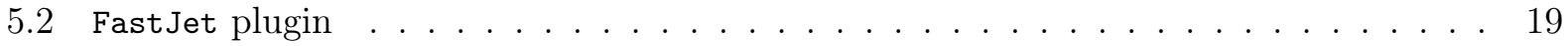

5.3 Jet and Template Moments . . . . . . . . . . . . . . . . 20

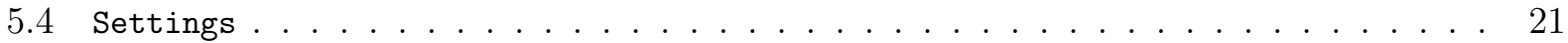

A Properties of Templates $\quad 22$

A.1 The case of 2 -body templates $\ldots \ldots \ldots \ldots \ldots \ldots$

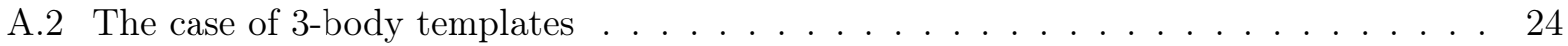

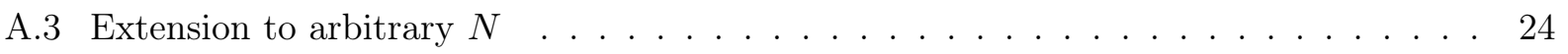

B Substructure and jet shapes 
C.1 Classes . . . . . . . . . . . . . . . . . . . . . . . 26

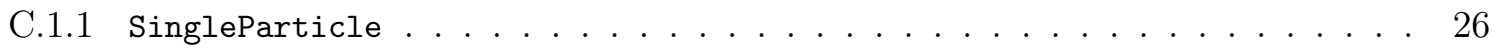

C.1.2 MeasureFunctor . . . . . . . . . . . . . . . . . 27

C.1.3 DefaultMeasure . . . . . . . . . . . . . . . . . 28

C.1.4 GaussianMeasure . . . . . . . . . . . . . . . . . 28

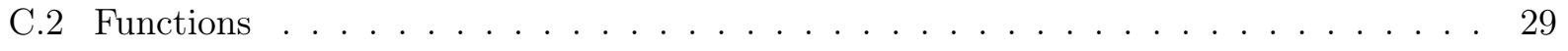

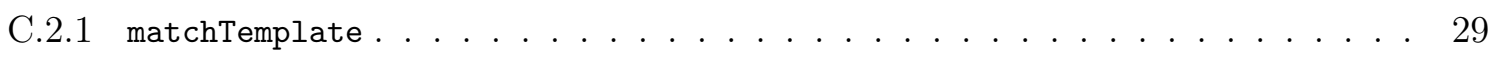

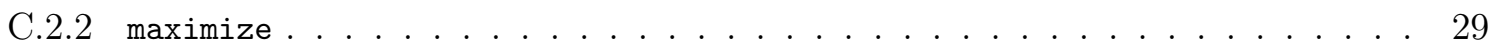

C.2.3 ConvertToPseudoJet . . . . . . . . . . . . . . . . . . . . 29

C.2.4 ConvertToMat . . . . . . . . . . . . . . . . 30

C.2.5 overlapDistance . . . . . . . . . . . . . . . . . 30

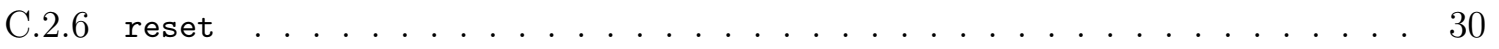

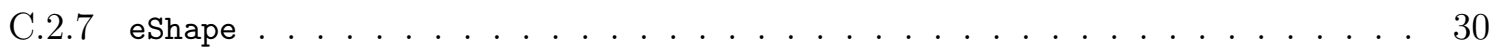

\section{Introduction}

Algorithms for tagging of boosted objects necessarily exploit observables sensitive to parton shower history, including color flow and hadronization. Most jet sub-structure methods can be characterized as jet de-clustering and re-clustering algorithms, with a common feature that they perform the analysis on the entire jet, after showering and hadronization. (see Refs. 11 19] for a review).

Tracking the parton shower history from physical final states to the hard-parton subprocesses often becomes rather involved as the number of QCD emissions is typically very large. Details of hadronization only further complicate the analysis. The Template Overlap Method [20 23], aims to bridge the gap between energy flow of observed jets and partonic configurations calculated at fixed order perturbation theory. The method allows for subjet identification in an infrared-safe way, by providing a mapping between energy-unweighted variables and the template that defines the energy flow distribution.

The primary intended use of the TemplateTAGger package is the analysis of jet substructure in High Energy Physics collider data. TemplateTagger allows the user to design a custom boosted jet analysis for a variety of scenarios by using the same basic three-stage approach. First, the user generates sets (or "catalogs") of templates by scanning over a phase space of the parton decay daughters of a massive particle of mass $M$ and transverse momentum $p_{T}$. Second, the 
TEMPlATETAGGER code performs template matching on an event-by-event basis whereby candidate signal jets are located in the $\eta, \phi, p_{T}$ space. Finally, the events are tagged using best matched templates (peak templates) as approximate subjet locations. The overlap approach has several important features:

- TemplateTagger is model independent. The user is required to define a template model, while the code will efficiently search for the matching subjet-like structures in the jet energy flow patterns.

- The pattern recognition based approach permits an efficient way of determining the jet topology while at the same time taking into account the event kinematics (jet mass, subjet asymmetry, etc.).

- TemplateTagger tools can be used to preserve as much energy flow information as possible, which is particularly useful for events where the energy distribution is all that is available. This information is presented in a well-organized form convenient for a detailed analysis of jet substructure.

- Peak templates have well defined jet shapes insensitive or weakly sensitive to pileup and underlying event.

TemplateTagger is a $\mathrm{C}++$ library which provides basic implementation of the Template Overlap Method for jet substructure. We designed the code around the FASTJET 24 package of jet algorithms with the aim at easy implementation into existing jet analysis tools. TEMPLATETAGger is also a testbed containing programs and routines for generating template data sets and collecting and analyzing statistics on the performance of Template Overlap and jet shapes. The package can be downloaded from http://tom.hepforge.org/.

In addition to overlap analysis, TemplateTAGger provides the necessary tools to analyze a boosted jet using observables constructed out of best matched templates. We provide implementations of various template-based jet shapes and energy flow observables such as Template Planar Flow, Template Angularity, etc.

This manual describes how to download and install TemplateTAGger, how to use the main libraries, as well as how to change its configuration for different overlap measures and template catalogs. Finally, the manual shows how to use the sample programs for testing purposes and basic data analysis. In section 2, we briefly introduce the physics behind the commands of TEMPLATETAGGER. We give a short description of the TEMPlateTAGger code structure in section 3. Section 4 lists several possibilities for further extensions of the program. Appendices A and B discuss boost-invariant implementations of the template generation and various jet shape observables. Appendix $\mathrm{C}$ contains the detailed syntax and functionality of all relevant internal methods.

\section{Physics Overview}

The current version of TEMPlateTAGGeR allows the user to study the substructure of massive high- $p_{T}$ jets for various models. The user defines a model by specifying a catalog of partonic decay 
configurations, labelled as $f$, which are taken to represent the decays of a heavy particle of mass $M$ at a given $p_{T}$. In addition, one has to specify a functional measure to quantify agreement between the energy flow of a jet and the flow of each template. For each jet candidate, the overlap function is defined as

$$
O v_{N}=\max _{\{f\}} \exp \left[-\sum_{a=1}^{N} \frac{1}{\sigma_{a}^{2}}\left(\epsilon p_{T, a}-\sum_{i \in j} p_{T, i} F\left(\hat{n}_{i}, \hat{n}_{a}^{(f)}\right)\right)^{2}\right]
$$

where $\{f\}$ is the set of templates defined for the given jet $p_{T}, p_{T, a}$ are the transverse momenta of the heavy particle (or resonance) decay daughters for the given template, $p_{T, i}$ is the $p_{T}$ of the $i^{\text {th }}$ jet constituent (or calorimeter tower, topocluster, etc.). The parameter $\epsilon$ serves to correct for the energy emitted outside the template subcone. The first sum is over the $N$ partons in the template and the second sum is over jet constituents. The kernel functions $F\left(\hat{n}, \hat{n}_{a}^{(f)}\right)$ restrict the angular sums to (nonintersecting) regions surrounding each of the template momenta. We provide two concrete implementations of kernels: a Gaussian around each of the directions of the template momenta with normalization $F\left(0, \hat{n}_{a}^{(f)}\right)=1$,

$$
F\left(\hat{n}_{i}, \hat{n}_{a}^{(f)}\right)=\exp \left[-(\Delta R)^{2} /\left(2 \omega_{a}^{2}\right)\right]
$$

and a normalized step function that is nonzero only in definite, non-overlapping, angular regions around the directions of the template momenta $p_{i}$,

$$
F\left(\hat{n}_{i}, \hat{n}_{a}^{(f)}\right)= \begin{cases}1 & \text { if } \Delta R<R_{a} \\ 0 & \text { otherwise }\end{cases}
$$

where $\Delta R$ is the plain distance in the $(\eta, \phi)$ plane. The parameters $\omega_{a}\left(R_{a}\right)$ determine the radial scale of the template subjet. Together with the energy resolution scale $\sigma_{a}$, these are the only tunable parameters of the model. A few possible strategies to determine the optimal values of $\sigma_{a}$ and $R_{a}$ are as follows:

- Choose the best parameters according to the tagging efficiency and background rejection (e.g. fix the efficiency and vary the sub cone size to achieve best background rejection power). Use the same parameters in every event.

- For each event, make a choice of parameters for which the overlap is maximized. Estimate the stability of the configuration.

- Choose the parameters separately for each template, e.g. using a $p_{T}$-dependent scale for template matching.

Template overlap provides a mapping of final states $j$ to partonic configurations $f[j]$ at any given order. The best matched template $f[j]$ can be used to characterize the energy flow of the jet, giving additional information on the likelihood that the event is signal or background. Furthermore, we can derive additional jet shape information out of $f[j]$ to further increase the rejection power of the method.

It is important to realize that other functional measures and kernel functions can easily be implemented, and we encourage the reader to explore them. The choice of template parameters, 
kernels and functional measures is largely dependent on the application of Template Overlap and has to be determined on a case by case basis. The same is true for the template generation. For instance, a boosted Higgs analysis benefits from the use of both the two-body and three-body overlap analysis, whereas two-body overlap could be of little use in a boosted top analysis.

\section{Template Overlap in a Pileup Environment}

\subsection{Effects of Pileup on Peak Overlap}

The high luminosity environment characteristic of the LHC provides an obstacle for all jet observables, with the recently finished $\sqrt{s}=8 \mathrm{TeV}$ run resulting in stunning $\left\langle N_{v t x}\right\rangle=20$ interactions per bunch crossing. Jet mass and transverse momentum as well as most jet substructure observables (see Ref. 25 for instance) can be severely affected by pileup, prompting a need for various pileup subtraction and correction techniques.

One of the valuable features of the Template Overlap Method is that it is weakly sensitive to pileup, even in a high pileup environment. There are three main reasons for this:

1. Template Overlap Method is designed to detect large "spikes" in energy deposition, characteristic of the hard radiation.

2. The peak overlap is sensitive only to the energy flow deposited within a template sub cone of radius $R_{a}$. To first order approximation, the effects of pileup on the value of overlap should be proportional to the area of the template sub-cones 1 .

3. The typically small template sub-cone (relative to the jet cone size) protects the Template Overlap method from a large pileup contamination.

Figure 1 shows an example of the effect of $\left\langle N_{v t x}\right\rangle=20$ pileup interactions on the distributions of three body peak overlap of boosted top jets. The distributions remain largely unaffected with ramifications of pileup resulting in $10 \%$ effects $[26]$. The same is true for other template observables 26].

\subsection{Using Multiple Template $p_{T}$ bins in a High Pileup Environment}

When running the overlap analysis, it is often beneficial to use several sets of template libraries, each one with a different template $p_{T}$ in order to better cover the $p_{T}$ range of the signal region. In a no-pileup environment, selecting the appropriate template $p_{T}$ bin is trivial, whereby the transverse momentum of the fat jet determines the template appropriate template $p_{T}$. Such a method is not

\footnotetext{
${ }^{1}$ For instance, consider a boosted Higgs jet clustered with a radius $R=1.0$ and analyzed with a two body template of radius $R_{a}=0.1$. The relative pileup contribution to the overlap compared to the jet $p_{T}$ will be of the order $O\left(R_{a}^{2} / R^{2}\right)=0.01$.
} 


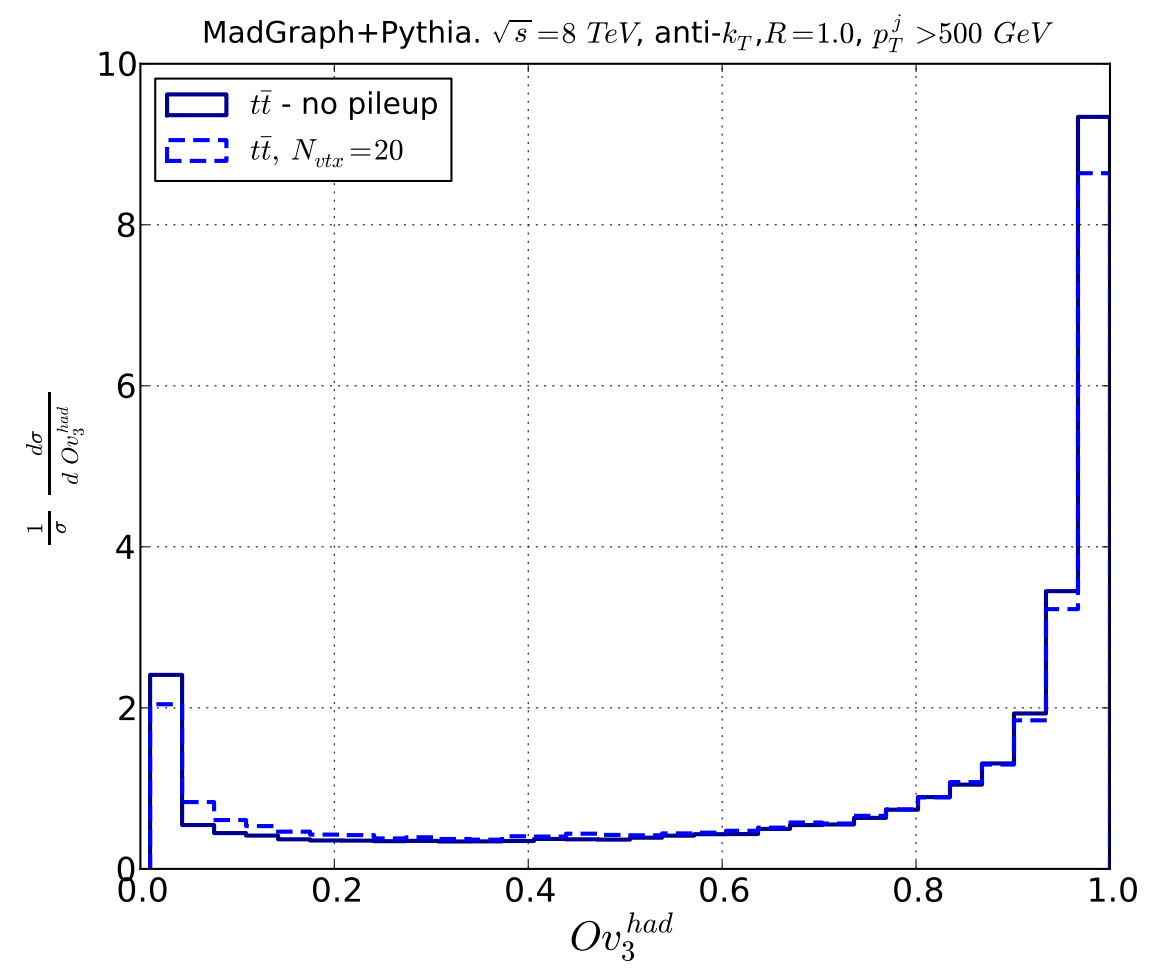

Figure 1: Effects of pileup on a three body peak overlap distribution of boosted top jets. The solid line shows events with no pileup, while the dashed line shows events $\left\langle N_{v t x}\right\rangle=20$ interactions per bunch crossing. 
always fitting in the presence of pileup as the $p_{T}$ of the fat jet is shifted to higher values, thus not accurately representing the $p_{T}$ of the underlying heavy particle of interest. There are two general ways to work around this issue without requiring pileup subtraction/correction methods:

1. Analyze each jet with every template library. The overlap analysis, by design, attempts to match the kinematics of the template to the kinematics of the hard part of the fat jet. Thus, the template library which gives the highest overlap will be the one with the $p_{T}$ closest to the transverse momentum of the hard part of the fat jet. This method is useful when analyzing events in which only jets are present. For instance, boosted $t \bar{t}$ events in which both tops decay hadronically. Notice however that this method also increases the computing time by a factor of roughly the number of template $p_{T}$ bins.

2. Use an observable which is less sensitive to pileup and is correlated with the fat-jet $p_{T}$. For instance, consider $Z h$ events in which $Z$ decays leptonically and Higgs decays to $b \bar{b}$. In the transverse plane, the Higgs and the $Z$ are emitted back to back, yielding $p_{T}^{l_{1}}+p_{T}^{l_{2}} \approx p_{T}^{h}$, but with the major difference that the $p_{T}$ of the leptons is almost unaffected by pileup. Thus, one can use the transverse momentum of the $Z$ to determine which template $p_{T}$ bin to use for the overlap analysis. Ref. [26] employs a similar method for the analysis of $W h$ events with large missing $E_{T}$.

\section{Program Structure and Use}

We proceed to discuss the installation of TEMPLATETAGger and execution of the example code. We also discuss the general structure of the code, for the benefit of the user who might wish to read or modify the source code.

\subsection{Installation}

TemplateTagger runs on any architecture with a modern $\mathrm{C}++$ compiler such as $\mathrm{g}^{++}$and an installation of FASTJET $[24$. For the convenience of Unix and Mac OS X users, we provide Makefile scripts. TemplateTagGer depends on FAstJet for finding jets and uses the internal FASTJET classes for implementation of basic relativistic kinematics. The current version of TemplateTaGger requires FASTJET version 3.0 or higher. To use the Makefile provided with the code, simply add the location where FASTJET is installed, so fastjet-config can be found.

To install and run the TemplateTagaer follow these steps 2 ;

1. In a web browser, navigate to

http://www.hepforge.org/archive/tom/

2. Download the (current) source tar-ball and extract it by typing:

\footnotetext{
${ }^{2}$ The following instructions are only for a Unix based operating system such as Linux or Mac OS X.
} 
tar -xvf TemplateOverlap-X.Y.Z.tar.gz

and replacing X.Y.Z with the appropriate version number (currently 1.0.0). This will create a new subdirectory TemplateOverlap-X.Y.Z where all the TEMPLATETAGGER source files are now ready and unpacked.

3. Move to the resulting directory (cd TemplateOverlap-X.Y.Z) and compile one of the examples

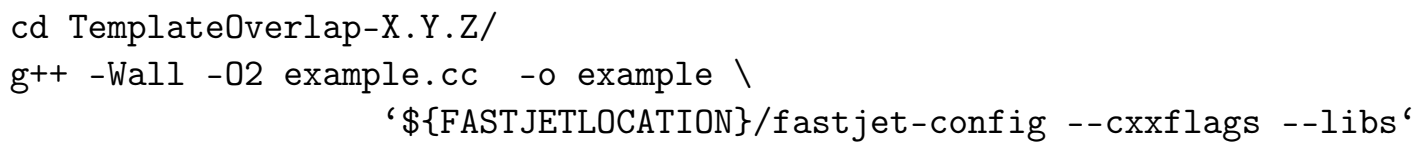

The code can also be compiled with the provided Makefile in environments where make is available.

4. The previous step compiles the example program which illustrates the basic functionality of the TemplateTagger. The program reads a test event file jet.dat and a template file template2b.dat. To execute the test program, type

. /example jet.dat template2b.dat

The example code will write output to the terminal, stdout, and should read:

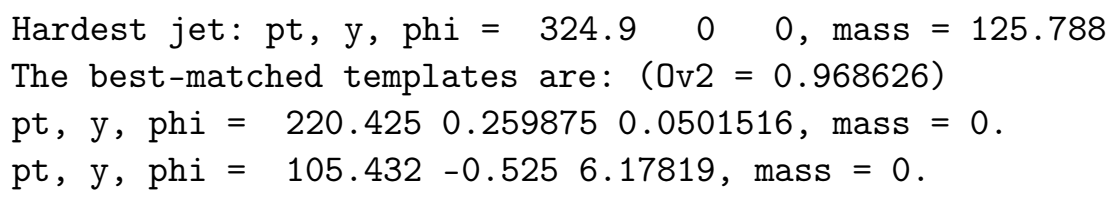

Now that you have successfully compiled and ran the example application (and perhaps even compiled and run it), we can proceed with a more detailed discussion of the TEMPLATETAGGER algorithm and structure. The following explanation will provide you with a basic understanding of the functionality of the underlying TemplateTAGger code.

\subsection{The Algorithm}

The core of the TEMPLATETAGGER package is a numerical implementation of the template matching algorithm of Eq. 1. The two primary components of every template matching process are:

- Source event $(j)$ : A jet containing full information about the constituents or calorimeter energy deposition.

- Template $(f)$ : A signal template which serves to construct a comparative measure.

The goal of Template Overlap analysis is to identify events $j$ with a good to templates $f$. Events with high match have a higher likelihood of being signal. The TEMPLATETAGGER package performs the analysis in a sequence general enough to be applicable in a wide variety of HEP analyses: 
1. Generate sets (or "catalogs") of large number of $N$-body templates which uniformly cover the phase space of a massive particle decay of mass $M$ at a given $p_{T}$. We suggest that templates be generated in the lab frame by solving all the available kinematical constraints, as in Appendix A. For a realistic analysis, it is usually necessary to generate several sets of templates for different values of $p_{T}$ and dynamically determine which template set is appropriate based on jet $p_{T}$. 3 Alternatively, templates can be generated in the rest frame of the event and boosted to the lab frame on an event-by-event basis. Note however, that this method comes with a significant increase in computation load, as millions of four momenta will typically have to be boosted for each event.

2. For each template, calculate a measure $d(j, f)$ to quantify the match (how similar the energy flow of the template is to that particular region in the flow of the observed event) of the template and the event

$$
d(j, f)=\exp \left[-\sum_{a=1}^{N} \frac{1}{2 \sigma_{a}^{2}}\left(\sum_{i \in \Omega} E_{i}^{(j)} F_{N}(\Omega, r)-E_{a}^{(f)}\right)^{2}\right],
$$

where $F_{N}(\Omega, r)$ is a kernel function and $r$ is the resolution scale parameter which determines the width and the shape of the kernel.

3. For each template $f$ and event $j$, store the measure $d(f, j)$ in the result matrix $\mathbf{R}$. The result matrix is analogous to the output of many image pattern recognition algorithms. Fig. 2 shows an example. The points represent a complete template set at a fixed $p_{T}$ and $M$ of a two body boosted Higgs decay. The color map represents the value of $d(f, j)$ for each template state. The regions of high $d(f, j)$ are where most of the event $p_{T}$ was deposited.

4. For every event $j$, find the maximum value

$$
O v_{N}=\max _{\{f\}} d(j, f)
$$

where $f$ refers to maximizing over the entire set of templates. We refer to $O v_{N}$ as the "peak overlap." Similarly, we refer to the template $f_{\max }$ which maximizes $d(f, j)$ as the peak template.

5. The previous three steps are repeated as many times as necessary using different values of $N$, e.g. for a boosted Higgs, $N=2,3$. Fig. 3 shows a typical matching process for a Higgs jet with $p_{T}=300 \mathrm{GeV}$ analyzed with both 2- and 3-particle templates.

\subsection{Efficient Generation of Template Libraries}

The TemplateTagger package provides routines which generate template libraries for a given model. The $N$-particle template libraries in the $\eta-\phi$ space can be represented using $3 N$-dimensional tables with equidistant grids in the $\eta, \phi$, and $p_{T}$ variables. The construction of such tables would typically proceed with the help of Monte Carlo data to determine the size of $p_{T}$ steps and the

\footnotetext{
${ }^{3}$ Choosing a template set based on jet $p_{T}$ could be inappropriate in a high pileup environment. See Section 3 for more detail.
} 


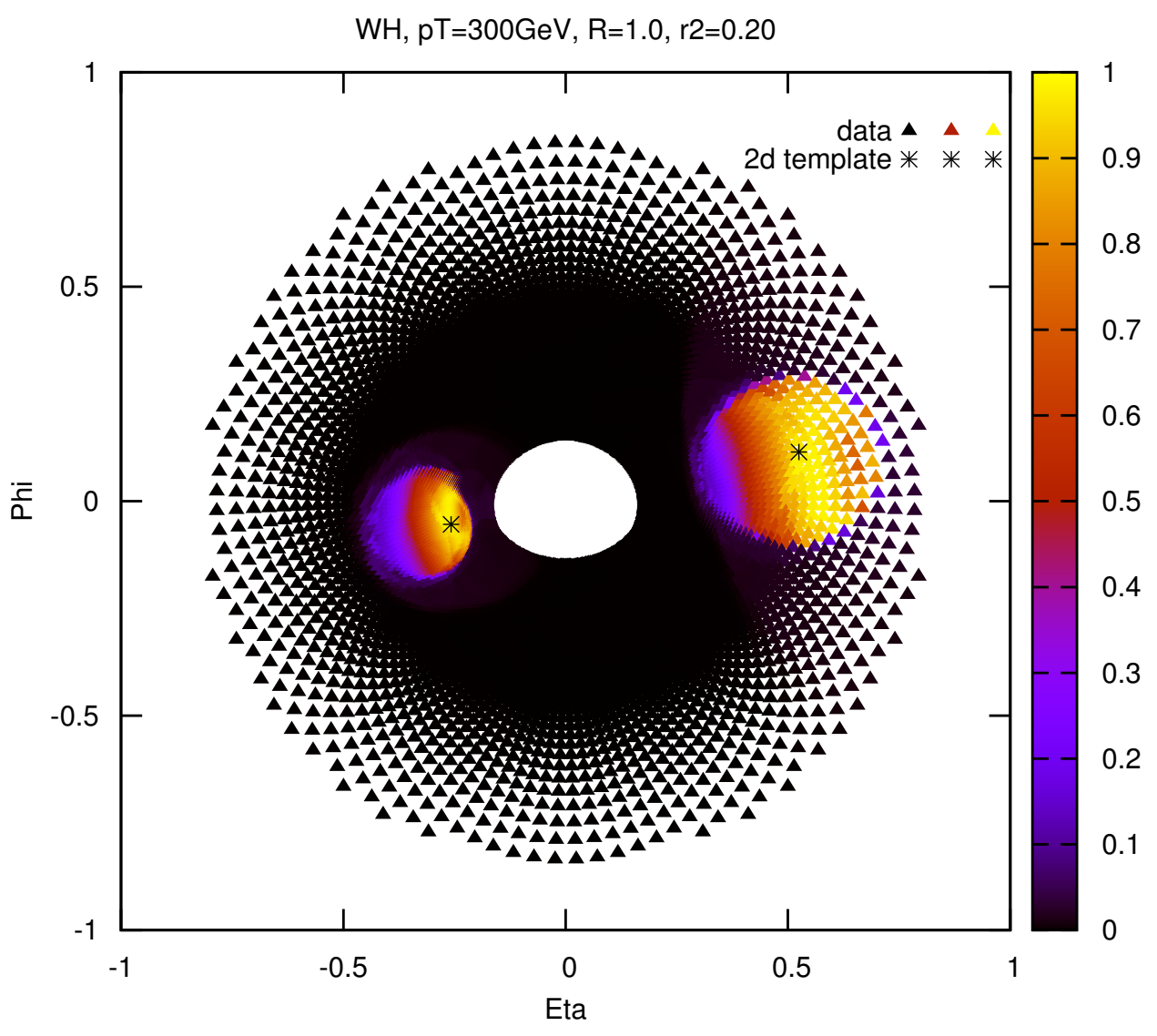

Figure 2: Energy flow reconstruction from 2-particle templates for a single boosted Higgs event. The points show angular positions of a highest $p_{T}$ template parton (two-particle templates). Note that the other parton is uniquely determined by energy conservation. The color map shows the overlap score of the template parton at various positions in $(\eta, \phi)$. The region around $\eta=\phi=0$ is not covered due to the kinematic constraint of $\Delta R_{b \bar{b}}>2 m_{h} / p_{T}^{h}$. 


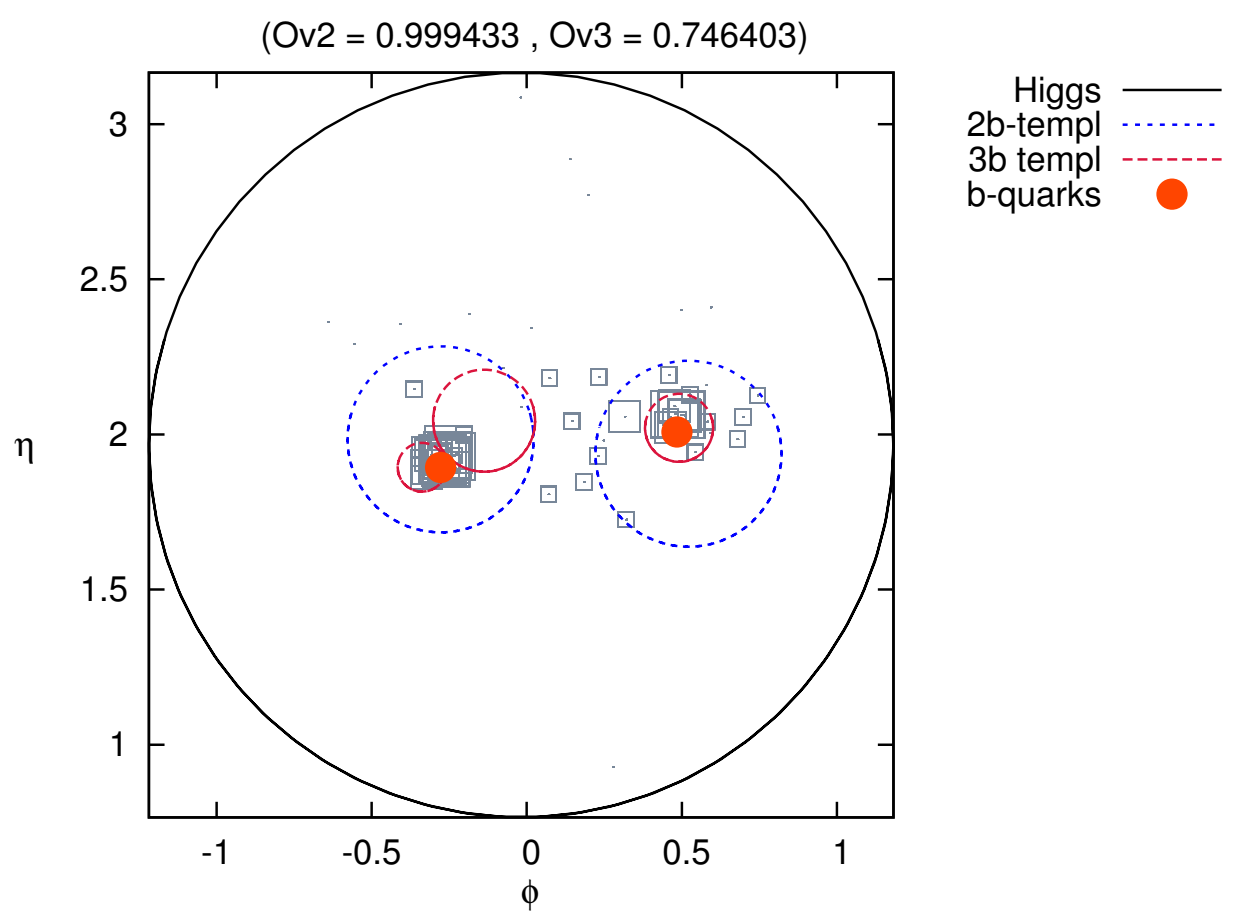

Figure 3: Event displays for a typical Higgs jet with invariant mass near $125 \mathrm{GeV}$. The blue and red circles indicate the region spanned by the best matched templates with $N=2,3$, respectively, using CONE and DefaultMeasure. In this and subsequent event displays, the particles are shown in grey cells of variable size, and the marker area for each cell is proportional its scalar transverse momentum. The solid red dots are the positions of $b$-quarks in the hard process.

minimum number of templates required to maximize signal efficiency while maintaining sufficient background rejection power.

The function TemplateBuilder in build_template.cc contains the full specification of how to carry out the generation of templates in the lab frame. According to the method described in Appendix A, a "template definition" should include parameters such as template $p_{T}$, mass and jet cone radius $R$ as well as the number of template patrons $N$. The function call is

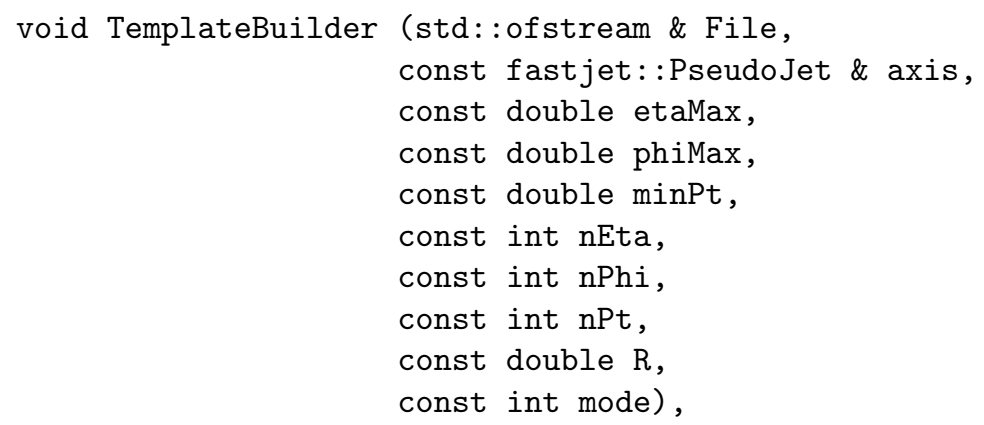

where File is the output file into which the code writes the template catalog and axis is the four 
vector of the event jet axis, providing relevant parameters like template mass and $p_{T}$. etaMax and phiMax are the maximum value of $\eta$ and $\phi$ of a template parton relative to the jet axis, while minPt is the minimum $p_{T}$ of a template parton (for infra-red safety). nEta, nPhi and $\mathrm{nPt}$ are the number of steps in $\eta, \phi$ and $p_{T}$ respectively for the template generation. $\mathrm{R}$ is the anti- $k_{T}$ jet cone used to cluster the template patrons. If the patrons can not be clustered into a jet of radius $\mathrm{R}$, the template is rejected. Finally, mode is one of the entries of the enumerated TemplateModel:

enum TemplateModel \{TOP, HIGGS2, HIGGS3, ...\};

The current implementation of TEMPLATETAGGER contains three default modes:

- TOP: Three body $t$ decay. The top template model generates template states to cover threeparticle phase space for top decay, $t \rightarrow b+W \rightarrow b+q+\bar{q}$, with the constraint $\left(p_{q}+p_{\bar{q}}\right)^{2}=M_{W}^{2}$. To construct these states, the algorithm uses a sequential scan over four angles. We take these to be the rapidities and azimuthal angles that define the $b$ and a $W$ 's daughter in the lab frame, defined relative to the direction of the top jet axis.

- HIGGS2: Two-body Higgs decay. Two angles define the two-body state of the daughter particles. We choose these to be the rapidity and azimuthal angle of the first daughter in the lab frame defined relative to the Higgs direction.

- HIGGS3: Three-body Higgs decay. Four angles and one energy define the three-body state of the daugher particles. We take these to be the the rapidities and azimuthal angles that define the $b$ and a $\bar{b}$ directions in the lab frame, defined relative to the Higgs direction. The remaining variable is the $p_{T}$ of the leading parton.

The TemplateBuilder routine surveys the kinematically-allowed template configurations by fixing the total four momentum to axis and then taking possible values of energies $\left(p_{T, i}\right)$ and the angles $\left(\hat{p}_{i}\right)$ within the bounded interval as defined by $\eta_{\max }, \phi_{\max }$ and $p_{T}^{\min }$. The number of variables depends on the number of degrees of freedom of the configuration. The domain of the $3 N-4$ independent variables, $\hat{p}_{1}, \cdots, \hat{p}_{N-1}$ and $p_{T, 1}, \cdots p_{T, N-2}$, that define a template is divided into a uniform grid, according to a fixed interval, and the remaining transverse momentum $p_{T, N-1}$ is obtained by applying the restrictions of conservation of energy-momentum. The resulting groups which include negative $p_{T}$ are automatically discarded. An additional restriction of

$$
p_{N}=P-\sum_{i=1}^{N-1} p_{i}
$$

imposes the condition $P=\left(p_{T}, 0,0, E_{J}\right)$.

\subsection{Choosing the Kernel}

The optimal choice of the template matching kernel depends on the analysis strategy and the amount of information the user has about the signal and the background. A reasonable choice of the kernel width typically assumes at least some kinematic properties or jet shapes of the signal. In fact, 
optimal choice will be different for different signals. For example, an analysis searching for a Higgs decaying into $b \bar{b}$ pairs is likely to make very different assumptions about jet substructure from a data analysis which looks for $t \bar{t}$ events in the all-hadronic 6 -jet mode. It is thus both interesting and important to look at the substructure of a jet using a variety of kernels and kernel parameters. Table 1 lists the kernels available in the default implementation of TEMPLATETAGGER.

The kernel function $F(\Omega, f)$ should be a sufficiently smooth function of the angles for any template state $f$ in order to ensure infra-red safety. For instance, the kernel could be defined as a Gaussian around each of the template momenta, which we provide with the option GAUSSIAN. Alternatively, we may choose $F$ to the a normalized step function that is nonzero only in definite angular regions around the directions of the template momenta $p_{a}$. This is the default option in TemplateTagger and is implemented as an option CONE.

The TemplateTagger package also allows the user to choose from a variety of template matching strategies to fix the energy resolution scales. A fast and simple template matching can be performed using a single resolution scale (at one fixed cone radius or Gaussian width). This is the setting of the FIXED option and is the default option in TEMPLATETAGGER. Alternatively, a more sophisticated choice can also take into account more complex jet shapes. Indeed, the optimal width is not necessarily the same for every jet in an event, as low momentum subjets tend to have wider angular profiles. As an implementation of this feature, we propose varying cone schemes for template matching. The scheme is similar to the fixed cone scheme, except that we allow for different cone radii to be associated to each template particle.

Both the kernel and the energy resolution scale are set by variables in Table 1 .

\begin{tabular}{|c|c|c|}
\hline Enum & Default option & Alternate option \\
\hline Jet_shape_scheme & CONE & GAUSSIAN \\
\hline Resolution_scale_scheme & FIXED & VARIABLE \\
\hline
\end{tabular}

Table 1: Members of the Jet_shape_scheme and Resolution_scale_scheme enums which define the choice of kernel function.

\subsection{Finding the Best Matched Templates}

MatchingMethod class, the core of the TEMPLATETAGGER package, provides the implementation of the algorithm which is responsible for the Template Overlap analysis as a whole. The algorithm is designed to perform template matching at fixed order in perturbation theory, with no constraint on the maximum level of complexity of energy flow patterns, e.g. when templates contain an arbitrarily large number of particles. Most important part of the MatchingMethod declaration (in the "matching.hh" header file) is the constructor of the MatchingMethod class:

MatchingMethod(const string \& templateFile, Settings mySettings)

The constructor arguments have the following meaning:

- templatefile A string containing the name of the template-catalog file. 
- mySettings An object which collects several settings to use for template matching. See Section 5.4 for more details.

The simplest way of performing template matching with TEMPLATETAGGER consists in constructing an object of this class at the beginning of the data analysis code and then running its get0v() member function on each jet. Fig. 4 shows a schematic representation of the algorithm.

The MatchingMethod loads the template catalog from a file using the default constructor. The member function get0v then reads a fastjet: :PseudoJet and passes the input through a sequence of template matching functions which processes the following sequence:

- Load an input event and a test configuration (template).

- Perform a template matching procedure by using the TemplateOverlap function matchTemplate with either of the two matching methods described before.

- Normalize the output of the matching procedure so that unity means perfect match.

- Localize the template with the highest matching probability.

- Return the maximum value of overlap and best matched configuration (maximum overlap template).

The get0v function returns the results of the maximization procedure in the format of temple_t (defined in Section 5), where the first element of the ordered pair is the value of the overlap and the second one contains a vector of template four momenta. The user can pass the result of get0v to several Function0fPseudoJets which compute jet observables from the momenta in the best matched template. 


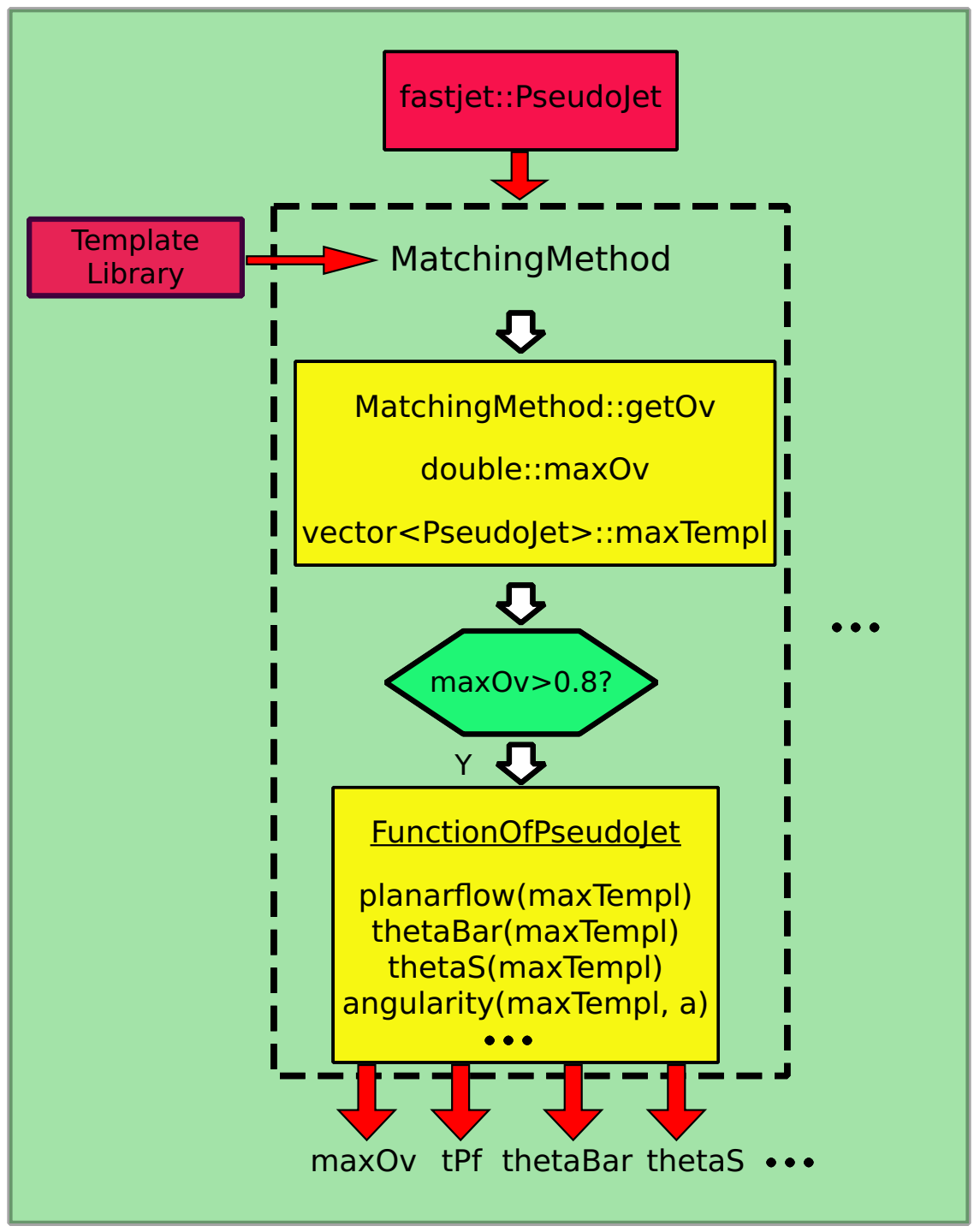

Figure 4: The structure of a typical TemplateTAgGer analysis. A MatchingMethod gets input from a template catalog file using the default constructor MatchingMethod: : MatchingMethod. The method getOv reads a fastjet: :PseudoJet and passes the input through a sequence of template matching functions that are run sequentially. The output of getov is then passed to several FunctionOfPseudoJets that compute jet observables based on angular distributions of the partons in the best matched template. 


\subsection{Sample Program}

An example program in short.cc provides the basic functionality of TEMPLATETAGGER. More specifically, the example shows how the MatchingMethod class can be used to perform basic substructure analysis with the Template Overlap method.

example requires only one input file: the template catalog. For the purpose of the example, consider a catalog of two-body templates stored in a file template2bf.dat. The command line call for example is:

./example template2bf.dat

Below, we give a detailed description of the relevant code snippets.

1. Include the appropriate header files. The core functionalities of TEMPLATETAGger are contained in the file matching.hh:

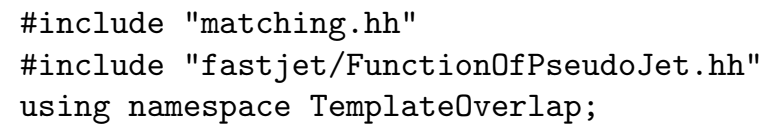

2. Declare run parameters, event and result containers, as well as the match method. Set the jet cone radius $R=1.0$ and the template sub-cone radius $r=0.2$ as well as $\sigma_{a}=p_{T}^{a} / 3$, where $p_{T}^{a}$ is the transverse momentum of the $a^{\text {th }}$ template parton. All settings are put into the Settings object:

/// Set Run Parameters

double $\mathrm{R}=1.2 ; / /$ anti-kt parameter

double $\mathrm{R} 2=0.40 ; / /$ template subcone radius

double sigma $=0.333 ; / /$ template Gaussian width

myParams = Settings $(R 2$, sigma, R);

3. Define the event to be analyzed. For the purpose of the example, we hard-coded an event.

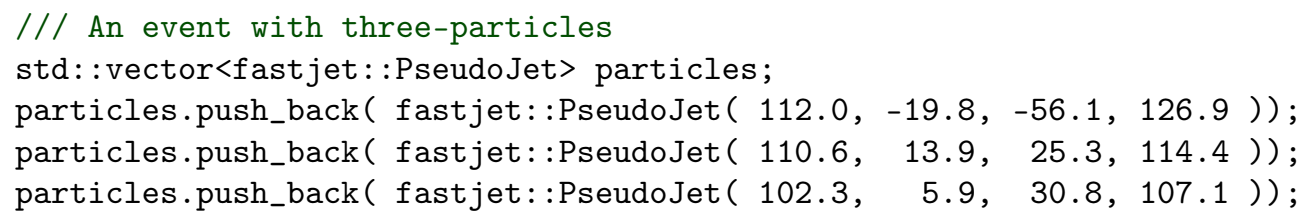

4. Define the template matching instance which uses the template catalog of template2bf.txt and the parameters stored in myParams:

/// Create an instance of MatchingMethod for the analysis.

/// The ctor also loads the templates

MatchingMethod myCone(argv[1], myParams); 
5. Analyze the event. temp.first contains the value of maximum overlap. temp. second contains the best matching template.

/// Find the best matched template

templ_t result $=$ myCone.get0v $($ jets $[0])$;

std: : vector<fastjet::PseudoJet> maxTempl = result.second;

double $\operatorname{maxOv}=$ result.first;

The getOv method performs the core functions of the Template Overlap Method. As such, we deem it important to provide a more detailed discussion of its structure.

1. Let us check the get0v function. First define the matching method and make a copy of the source event:

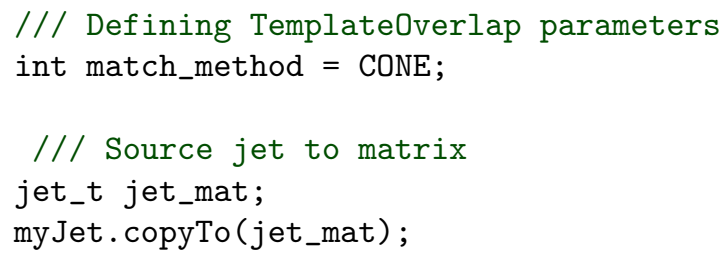

The jet_t typedef is a vector of particles, that collects basic kinematic information about the constituents in a jet, i.e. their $\eta, \phi$ and $p_{T}$.

2. Next, create the matrix that will store the results for each template location:

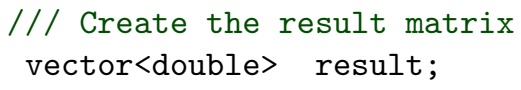

3. Use the TemplateOverlap function matchTemplate to search for matches between a template and a input event:

/// Do the matching and normalize

matchTemplate(jet_mat,_templates, result, match_method);

the arguments are naturally the input event $j$ (jet_mat), the templates $f$ (_templates), the result $\mathbf{R}$ (result), and the match_method

4. Use the TemplateOverlap function maximize to find the maximum overlaps (as well as their template directions) in the result array:

/// Localizing the best match with minMaxLoc

double maxVal; int maxLoc;

maximize (result, maxVal, maxLoc );

The arguments of the function are: 
- result: the source array.

- maxVal: variable to save the maximum value in the array, i.e. the maximum overlap.

- maxLoc: the point location of the maximum values in the array, i.e. the best match template.

5. Convert to PseudoJet and return the result:

vector $<$ PseudoJet $>$ peak_template $=$ ConvertToPseudoJet (_templates [_maxLoc], jet); return std::make_pair<double, vector<PseudoJet> >(_maxVal, peak_template);

In summary, the output of getov for any PseudoJet is the value of the overlap $O v_{N}$, and also the identity of the peak template as a vector of PseudoJets.

\section{Miscellaneous Tools}

\subsection{Data types}

We have defined two new data types, templ_t and jet_t for convenience. Here we briefly list the new data types for completeness and clarity, as they appear throughout the TEMPLATETAGGER code. We typically use templ_t to store the results of the overlap analysis for each event. The double value typically holds the maximum overlap, while the vector of PseudoJet holds the best matching template. The definitions are:

1. typedef std::pair<double, std: :vector<fastjet::PseudoJet $>>$ templ_t;

2. typedef std: :vector $<$ SingleParticle $>$ jet_t;

\subsection{FastJet plugin}

With the advent of FASTJET 3.0+, it has become straightforward to write wrappers for jet analysis tools around the suite of tools available in FASTJET. The FAST JET bare class FunctionOfPseudoJet<T> provides a common interface for jet measurements. For the convenience of the user, we provide a class Noverlap, defined in TemplateTagger.hh, which performs all functions to the TemplateTAGger code within the FASTJET framework. The class wraps the core Template Tagger code to provide the fastjet::FunctionOfPseudoJet interface for convenience in larger analyses. See matching.hh for definitions of $O v_{N}$ and the constructor options. The relevant methods of the class are:

1. Noverlap Noverlap(int $\mathrm{N}$, double sigma, double RO, const string \& templateFile) 
Constructor for the Noverlap class. N represents the order of the overlap analysis (two body, three body ...), sigma is the fraction of template parton $p_{T}^{a}$ used in $\sigma_{a}$. Ro is the jet cone radius and templateFile is the file containing the template catalog.

2. PseudoJet templ_t result (const PseudoJet\& jet) const

This function returns the results of the overlap analysis in the format of a pair of values, the maximum value of overlap and the best matched template. See the discussion of get0v for more information.

FASTJET $3.0+$ also provides a common base class for jet manipulation: Transformer. Transformers can remove particles, re-arrange substructure, or tag/reject jets. The class TemplateTagger in TemplateTagger.hh implements a generic Template Overlap code described in the previous sections.

The TemplateTagger class derives from Transformer, and can be constructed using a pointer to a Selector class derived from FunctionOfPseudoJet<templ_t $>$ which contains a value for the template kernel width, and the name of a file containing the catalog of templates. A simple example illustrates the implementation of TEMPlateTAGger within the FASTJET architecture:

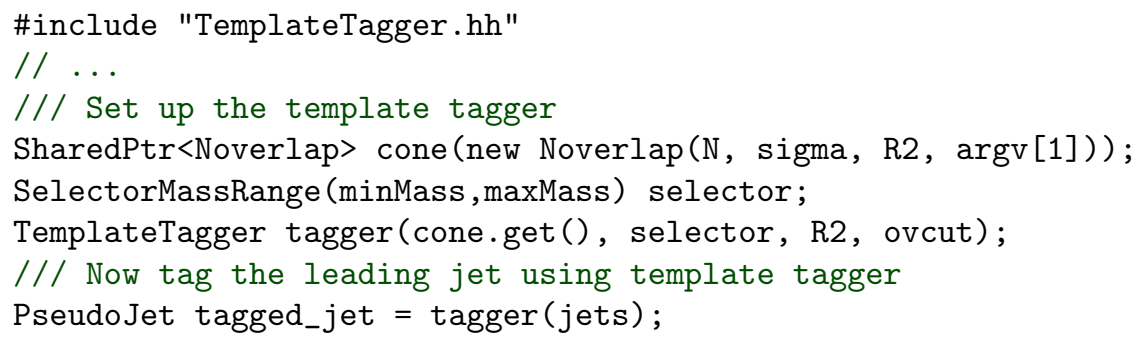

We adopt the convention that if a given jet does not satisfy $O v_{N}$ >ovcut the result of the transformer is a jet whose 4-momentum is zero. The pieces () of the resulting tagged jet correspond to the subjets that were associated to the best matched template:

std: : vector<fastjet: :PseudoJet> subjets = tagged.pieces ();

Additional structural information related to the value of the maximum overlap value and the best matched template is easily accessible. For instance:

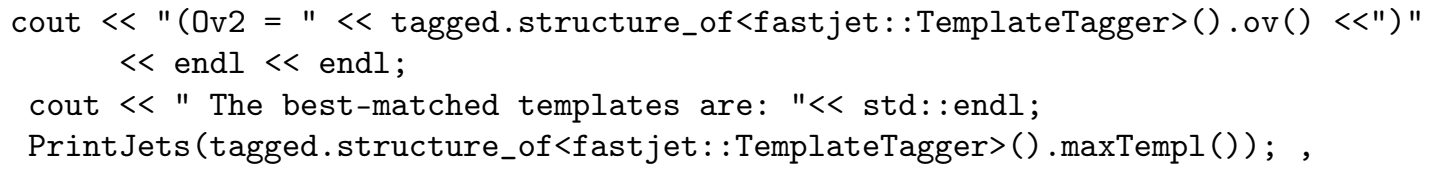

displays the value of maximum overlap and the four momenta of the best matched template.

\subsection{Jet and Template Moments}

TemplateTAgger allows a user to calculate additional substructure observables. The FASTJET 3 base class fastjet: :FunctionOfPseudoJet<double> provides a common interface for the calculation. The following FunctionOfPseudoJets are available ( all defined in TemplateTagger.hh): 
planarflow(): Calculates planar flow of the jet using longitudinally boost-invariant quantities.

angularity (int a): Calculates the angularity $\tau_{-a}$ of the jet.

$\operatorname{thetaS}()$ : Calculates the angle between the softest template particle and the jet axis.

thetaBar(): Calculates an energy-unweighted distance between the template particles.

Stretch(): Calculates the imbalance in $\Delta R$ between the peak two-body template and the two leading subjets inside the jet.

Area (): Calculates the template area, i.e. the area in $\eta-\phi$ space projected by the cones around the directions of the template particles.

Each function returns a double value. For a more detailed description of the above mentioned observables see Appendix B

\subsection{Settings}

The Settings class keeps track of all modes and parameters used during the jet clustering and template matching processes. As such, it serves all the MatchingMethod program elements from one central settings record. The user is allowed to access and change these settings to modify the template matching behavior. The complete list of methods and arguments is as follows.

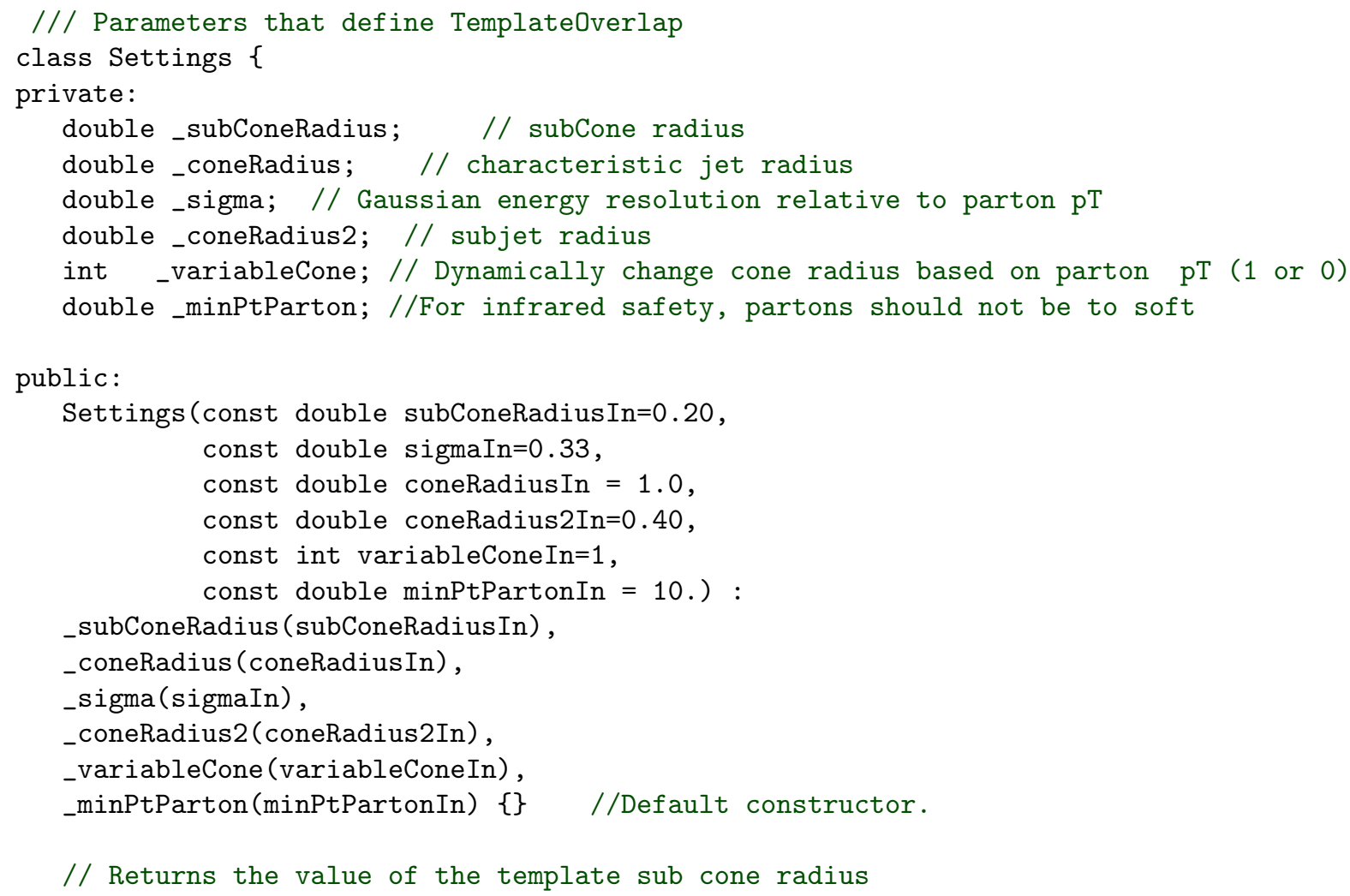




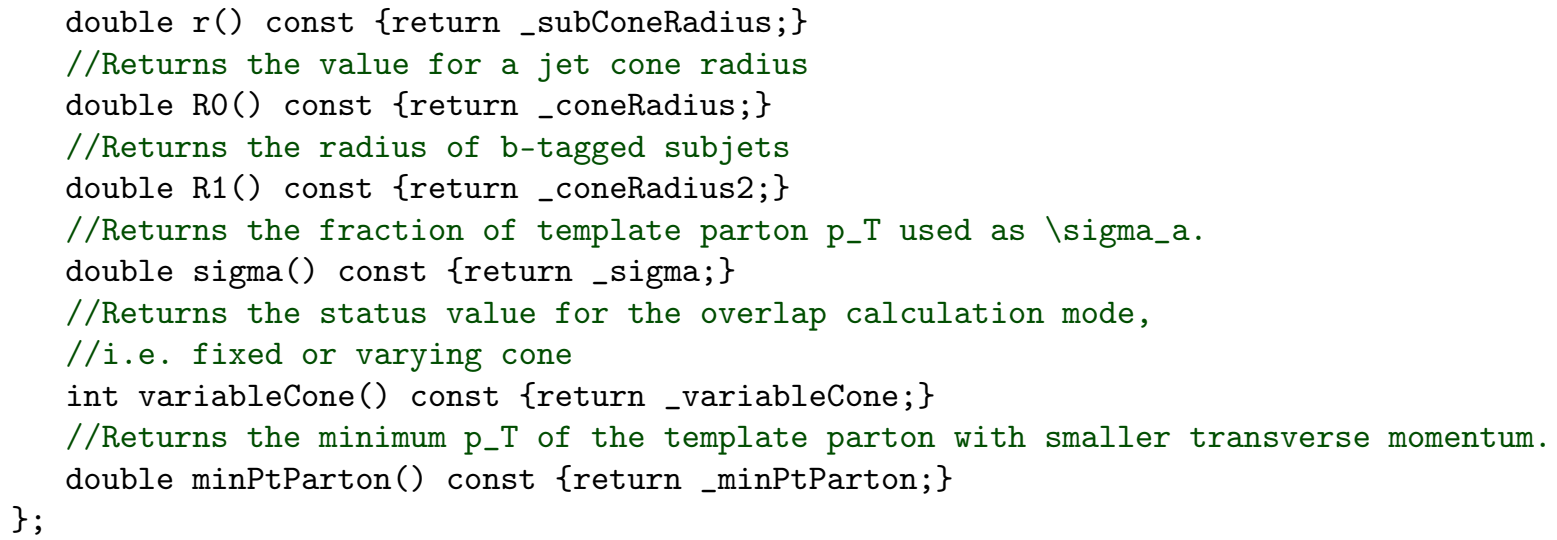

\section{Acknowledgments}

We thank Gilad Perez for introducing us to this topic and for a strong support in all stages of this project. We are very grateful to L. Levinson, P. Choukroun and the rest of the managers of Weizmann Institute physics computing farm for their flexibility and enormous help with large scale calculations. We thank S.J. Lee and R. Alon for the help with the early versions of the code and their suggestions and Gavin Salam for reading the code and sending valuable feedback. We also benefited greatly from discussions with P. Sinervo J. Winter, F. Spano, E. Duchovni and O. Silbert. Finally, we would like to thank the CERN Theory group for their hospitality.

\section{A Properties of Templates}

Template Overlap Method is a systematic framework aimed to identify kinematic characteristics of an boosted jet. A typical template configuration consists of a model template, $f$, calculated in perturbation theory, which describes a "prong-like" shape of the underlying hard subprocess of a jet. Template construction typically employs prior theoretical knowledge of the signal kinematics and dynamics, as well as possible experimental input. For instance, Higgs 2-particle templates are sets of 2 four momenta which satisfy kinematic constraints of a boosted Higgs decay etc.

The simplest template configurations are the ones describing the kinematics of two-body processes such as the decay of SM Higgs or $W / Z$ bosons into quark-antiquark pairs. These are easily dealt with by assuming the rest frame of the parent particle and producing two decay products with equal and opposite, isotropically-selected momenta and magnitude, subject to energy conservation. The problem of a $N$-body decay subtracts four constraints from the decay products' $3 N$ degrees of freedom: three for overall conservation of momentum and one for energy The final states can therefore be found on a $(3 N-4)$-dimensional manifold in the multi-particle phase space. Note that the dimensionality of the template space increases rapidly with additional patrons. For instance, the

\footnotetext{
${ }^{4}$ For our purpose, we ignore the color and spin degrees of freedom.
} 
two body templates require only two degrees of freedom, while a corresponding four body template space is already eight dimensional.

The question of which kinematic frame the templates should be generated in requires careful consideration. Authors of Ref. 27] argued that a search for the global maximum of $O v_{N}$ could be too computationally intensive. To improve the computation time, the template states were generated in the Higgs rest frame using a Monte Carlo routine, and then boosted into the lab frame. While this method worked sufficiently well for tagging a highly-boosted object(i.e. a $1 \mathrm{TeV}$ Higgs jet), it introduced residual algorithmic dependence and a certain sense of arbitrariness in the jet shape. At lower $p_{T}$ the Monte Carlo approach samples mainly the templates within the soft-collinear region, leaving other regions of phase space unpopulated. An enormous number of templates is required to adequately cover the phase space at $p_{T} \sim O(100 \mathrm{GeV})$, thus fully diminishing the motivation for a Monte-Carlo approach. The simplest and most robust choice is then to generate templates directly in the lab frame and then rotate them into the frame of the jet axis. The result is a well covered template phase space in all relevant boosted frames. In addition, the lab frame templates result in a significant decrease in computation time as a much smaller number of templates are needed.

We proceed to show how to generate the phase space for 2- and 3-parton final states as well as how to generalize the results to arbitrary $N$.

\section{A.1 The case of 2-body templates}

First, we summarize our notation and conventions. The model template consists of a set of four vectors, $p_{1}, \cdots, p_{N}$, on the hyperplane determined by the energy-momentum conservation,

$$
\sum_{i} p_{i}=P, P^{2}=M^{2}
$$

where $M, P$ are the mass and four momentum of a heavy boosted particle, i.e. the Higgs. For simplicity, we treat all template particles to be massless. We work in an $\left(\eta, \phi, p_{T}\right)$ space, where $\eta$ is pseudorapidity, $\phi$ azimuthal angle and $p_{T}$ transverse momentum. Without loss of generality, we can assume that the template points in the $x$ direction $(\eta=\phi=0)$. The templates are distributed according to

$$
p_{i}=p_{T, i}\left(\cos \phi_{i}, \sin \phi_{i}, \sinh \eta_{i}, \cosh \eta_{i}\right), i=1,2,3
$$

subject to the constraint

$$
\sum_{i=1}^{N} p_{i}=P=\left(p_{T}, 0,0, E_{J}\right)
$$

with $E_{J}=\sqrt{M^{2}+p_{T}^{2}}$. We find it useful to define unit vectors by

$$
\hat{p}_{i}=\left(\cos \phi_{i}, \sin \phi_{i}, \sinh \eta_{i}, \cosh \eta_{i}\right), i=1,2,
$$

so that $p_{i}=p_{T, i} \hat{p}_{i}$.

Phase space for the 2-body decay processes is characterized by particularly simple kinematic parameters. To illustrate, first note that the 2-particle templates are uniquely determined by one 
single four momentum, $p_{1}$ subject to the condition

$$
\left(P-p_{1}\right)^{2}=0
$$

Writing $p_{1}=p_{T, 1} \hat{p}_{1}$, we can solve for $p_{T, 1}$ in terms of the angles of the first parton

$$
p_{T, 1}=\frac{M^{2}}{2\left(P \cdot \hat{p}_{1}\right)} .
$$

We see that a 2-particle template is therefore completely determined in terms of the unit vector $\hat{p}_{1}$ as follows:

$$
\begin{aligned}
p_{1} & =\frac{M^{2}}{2\left(P \cdot \hat{p}_{1}\right)} \hat{p}_{1} \\
p_{2} & =P-p_{1} .
\end{aligned}
$$

Note that we can represent such a template as a point $(\hat{\eta}, \hat{\phi})$ in $\eta-\phi$ plane. These are the two degrees of freedom, in accordance with the general result that the dimensionality of the $N$ template space is $3 N-4$.

\section{A.2 The case of 3-body templates}

A space of five degrees of freedom allows for 3-particle templates to differ from one another in more than one way. The 3 -particle templates are determined by two four momenta, $p_{1}$ and $p_{2}$, subject to the constraint,

$$
\left(P-p_{1}-p_{2}\right)^{2}=0
$$

Using $p_{1}=p_{T, 1} \hat{p}_{1}$ and $p_{2}=p_{T, 2} \hat{p}_{2}$, we can solve for $p_{T, 2}$ in terms of the angles of first two partons and $p_{T, 1}$,

$$
p_{T, 2}=\frac{M^{2}-2 P \cdot p_{1}}{2\left(P \cdot \hat{p}_{2}-p_{1} \cdot \hat{p}_{2}\right)} .
$$

A general 3-particle template is then completely specified by $p_{T, 1}$ and two unit vectors (or, equivalently, four angles) $\hat{p}_{1}$ and $\hat{p}_{2}$.

\section{A.3 Extension to arbitrary $N$}

A generalization to an arbitrary number of particles is straight-forward. Proceeding as above, the $N$-particle templates are determined by $p_{1}, \cdots, p_{N-1}$ subject to the constraint,

$$
\left(P-\sum_{i=1}^{N-1} p_{i}\right)^{2}=0 .
$$


Using $p_{i}=p_{T, i} \hat{p}_{i}$, we can now solve for $p_{T, N-1}$ in terms of the $p_{1}, \cdots, p_{N-2}$ and $\hat{p}_{N-1}$,

$$
p_{T, N-1}=\frac{M^{2}+2 \sum_{i<j}^{N-2} p_{i} \cdot p_{j}-2 P \cdot \sum_{i}^{N-2} p_{i}}{2\left(\hat{p}_{N-1} \cdot P\right)-2 \hat{p}_{N-1} \cdot \sum_{i}^{N-2} p_{i}}
$$

For the special cases of $N=2$ and $N=3$, this formula reduces to the above results .

\section{B Substructure and jet shapes}

The TemplateTagger code contains implementations of several jet shape observables in addition to the Template Overlap Method. Jet shapes are inclusive, infrared-safe observables which are smooth functions of the energy distribution within jets. They are constructed as weighted sums over the four-momenta of the constituents of a jet and reveal details about its inner structure, shedding light on its partonic origin.

- A set of such jet shape observables is given by the class of angularities $\tau_{a}$ of a jet, defined by

$$
\tau_{a} \equiv \frac{1}{2 E_{J}} \sum_{i \in J}\left|\mathbf{p}_{\mathbf{T}}^{\mathbf{i}}\right| e^{-\eta_{i}(1-a)}
$$

where $a$ is a parameter taking values $-\infty<a<2$, the sum is over all the particles in the jet, $E_{J}$ is the jet energy, $\mathbf{p}_{T}$ is the transverse momentum relative to the jet direction, and $\eta=-\ln \tan \theta / 2$ is the pseudorapidity relative to the jet direction.

Angularities, $\tau_{a}$, are able to distinguish between QCD jets and other two-body decays. Almeida et al. 23] showed that the discriminating power of angularities is owned to the fact that the decays of color neutral objects are democratic, sharing energy symmetrically, whereas QCD events with same mass are typically asymmetric.

- Planar Flow $(P f)$ [28 30] is another useful jet substructure observable. We defined the default TemplateTagger implementation of $P f$ in terms of pseudorapidity $\eta=-\ln (\tan (\theta / 2))$, the azimuthal angle $\phi$ and the transverse momentum $p_{T}$ :

$$
P f=\frac{4 \operatorname{det} \mathbf{I}}{(\operatorname{tr} \mathbf{I})^{2}}
$$

where $\mathbf{I}$ is defined by,

$$
\mathbf{I}=\frac{1}{m_{J}} \sum_{i} p_{T}^{i}\left(\begin{array}{cc}
\left(\Delta \eta_{i}\right)^{2} & \Delta \eta_{i} \Delta \phi_{i} \\
\Delta \eta_{i} \Delta \phi_{i} & \left(\Delta \phi_{i}\right)^{2}
\end{array}\right)
$$

with $m_{J}$ the jet mass, $p_{T}^{i}$ is the transverse energy of particle $i$ in the jet. Here, $\left(\Delta \eta_{i}, \Delta \phi_{i}\right)=$ $\vec{c}_{i}-\vec{J}$, where $\vec{J}=\left(\eta_{J}, \phi_{J}\right)$ is the location of the jet and $\vec{c}_{i}$ is the position of a cell or particle with transverse momentum $p_{T}^{i}$. Notice that the $P f$ definition of Eq. 20 is invariant under boosts along the beam axis.

Planar flow describes the way energy is deposited on the plane transverse to the jet axis. It peaks at zero for linear energy depositions and is close to unity for uniform energy configurations. 
For instance, two-pronged jets, such as leading order QCD jets, are expected to leave two cores of energy resulting in average low planar values of planar flow. On the other hand, three-prong jets coming from hadronic decays of boosted tops, are expected to have a rather uniform planar flow distribution. Thus planar flow can be used to separate massive boosted QCD jets from top jets.

- Angular correlations of the template momenta which can otherwise be concealed in the numerical values of the peak overlap are of particular value. For instance, the angular distribution of a jet radiation can be measured with the variable $\bar{\theta}$, defined as

$$
\bar{\theta}=\sum_{i} \sin \Delta R_{i J}
$$

where $\Delta R_{i J}$ is the distance in the $\eta-\phi$ plane between the $i^{\text {th }}$ template momentum and the jet axis. When measured using three-boy templates, the variable $\bar{\theta}$ characterizes the difference in angular ordering in our peak templates between the signal and background. Notice that for highly boosted jets, the 2-body version of $\bar{\theta}$ simply reduces to the angle between the two templates.

- Template Stretch is a pileup insensitive observable sensitive to the mass difference between a jet and the peak template. First introduced in Ref. [26], template stretch is defined as:

$$
S_{b \bar{b}}^{(f)}=\frac{\Delta R_{b \bar{b}}}{\Delta R_{f}} .
$$

where $\Delta R_{f}$ is the distance between the peak two-body template momenta and $\Delta R_{b \bar{b}}$ is the distance between the two $b$-tagged sub-jets. A generalization of $S$ to non-b-tagged jets and other kinematic configurations is straightforward.

\section{TemplateTagger Classes and Commands}

\section{C.1 Classes}

\section{C.1.1 SingleParticle}

SingleParticle is a helper class for MatchingMethod whose aim is to contain the minimum information about a particle in an event or a template, mainly its energy (or transverse momentum), rapidity and azimuthal angle. When the particle is associated with a template, there are two variables that contain additional, non-kinematics information: the template parton's width parameter radius (i.e. radius of the template sub cone), and its energy resolution sigma.

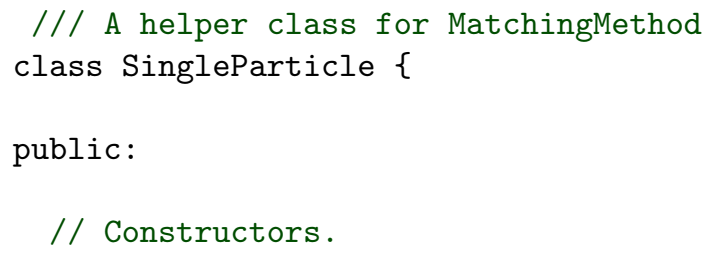




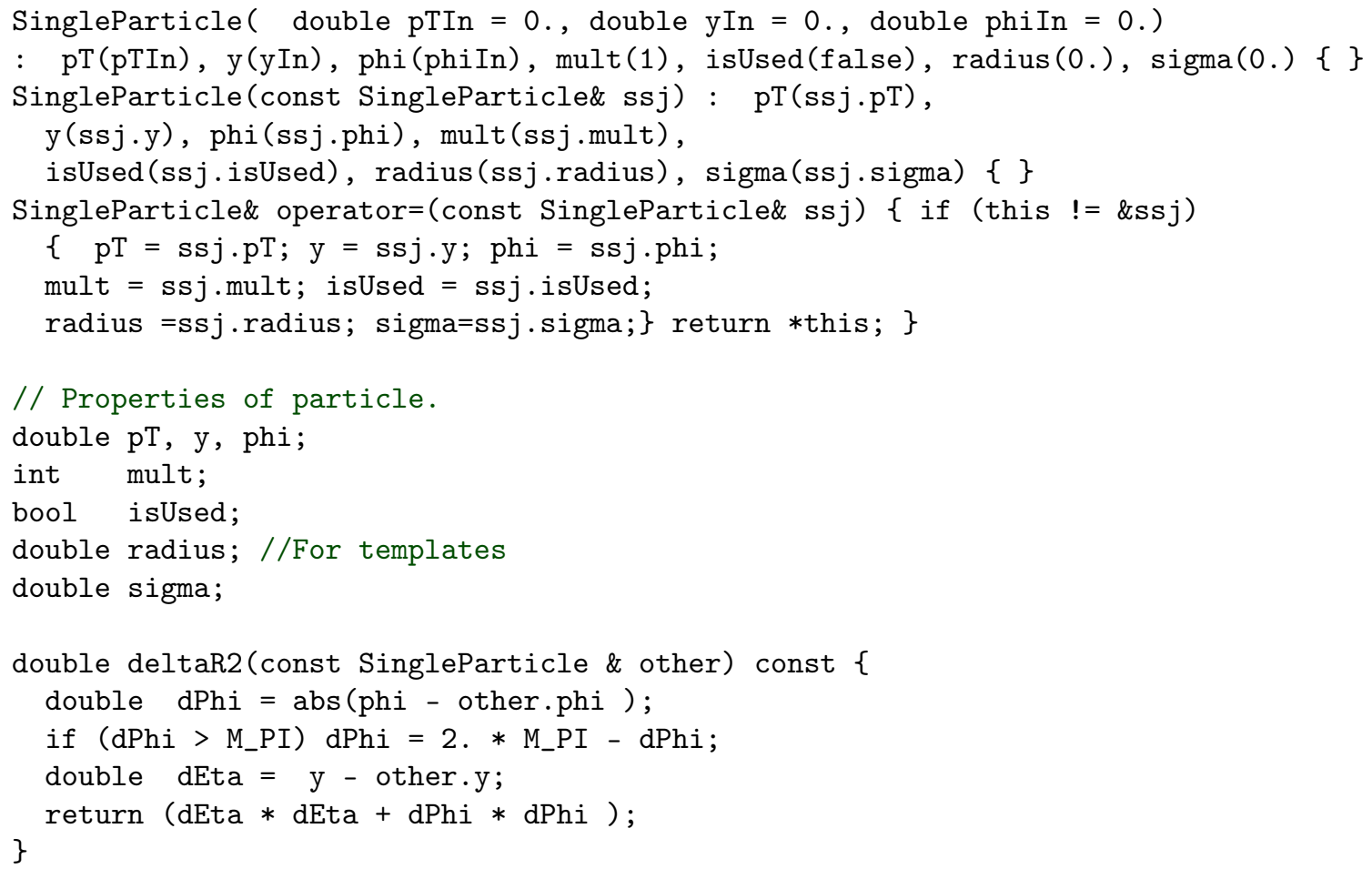

\section{C.1.2 MeasureFunctor}

TemplateTagger provides a bare class to define custom overlap functions: MeasureFunctor. This provides the user with some flexibility to specify different kernel functions or to build new ones for customized template analyses. The MeasureFunctor provides the minimum bare class from which other overlap functions can be derived. The most important part of the class declaration looks as follows:

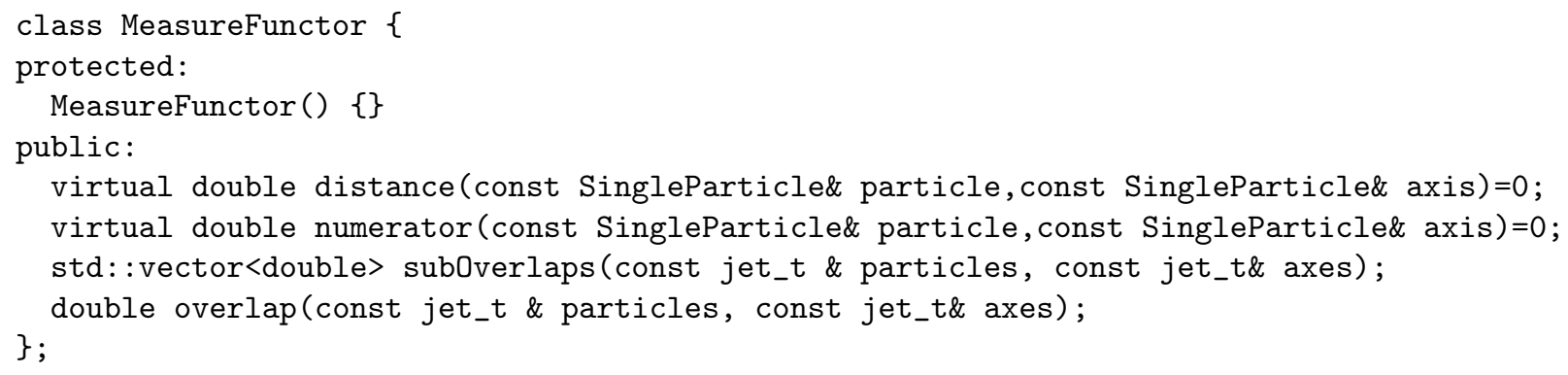




\section{C.1.3 DefaultMeasure}

The DefaultMeasure implements a cone-based template matching with the function

$$
F\left(\hat{n}_{i}, \hat{n}_{a}^{(f)}\right)=\left\{\begin{array}{ll}
1 & \text { if } \Delta R<R_{a} \\
0 & \text { otherwise }
\end{array},\right.
$$

By default, this function finds all particles within a cone of radius $R$ from the template parton and calculates the unweighted sum of the particles $p_{T}$. By default, a fixed cone radius $R$ is used. This can be modified via the Settings: : set_varying_cone(). This option corresponds to varying cone. Note that for the varying cone mode to work the user needs to specify a model for the cone scaling rule (energy profile). See eShape for more details.

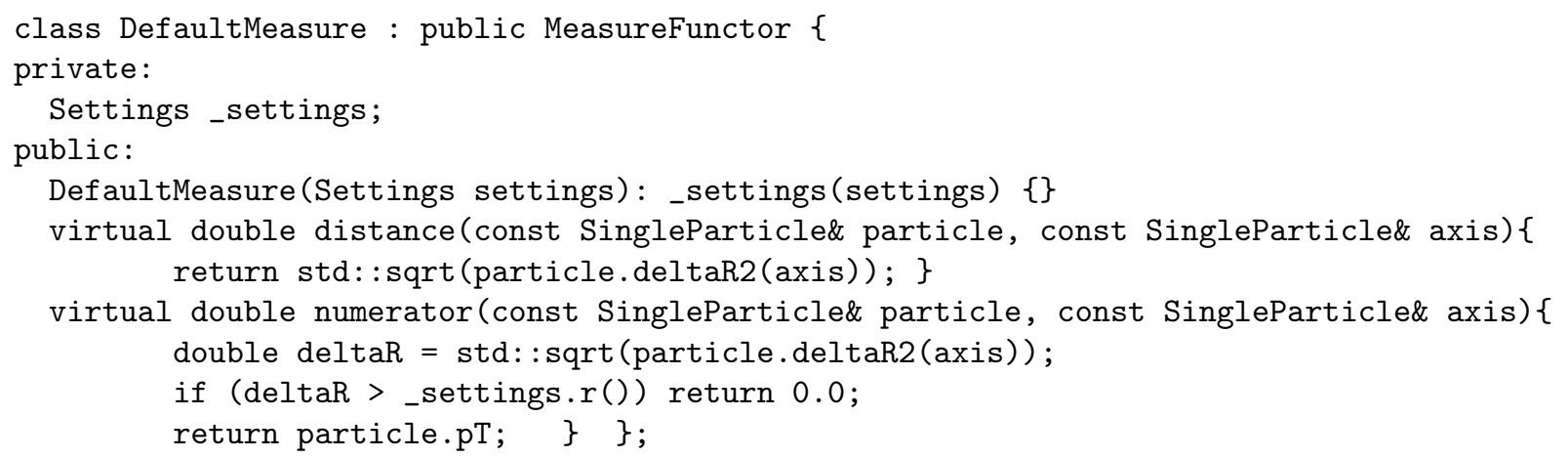

\section{C.1.4 GaussianMeasure}

Similar to DefaultMeasure, but uses a Gaussian kernel function

$$
F\left(\hat{n}_{i}, \hat{n}_{a}^{(f)}\right)=\exp \left[-(\Delta R)^{2} /\left(2 \omega_{a}^{2}\right)\right]
$$

to add the $p_{T}$ of each particle in a jet. It has two constructors with the same arguments as DefaultMeasure.

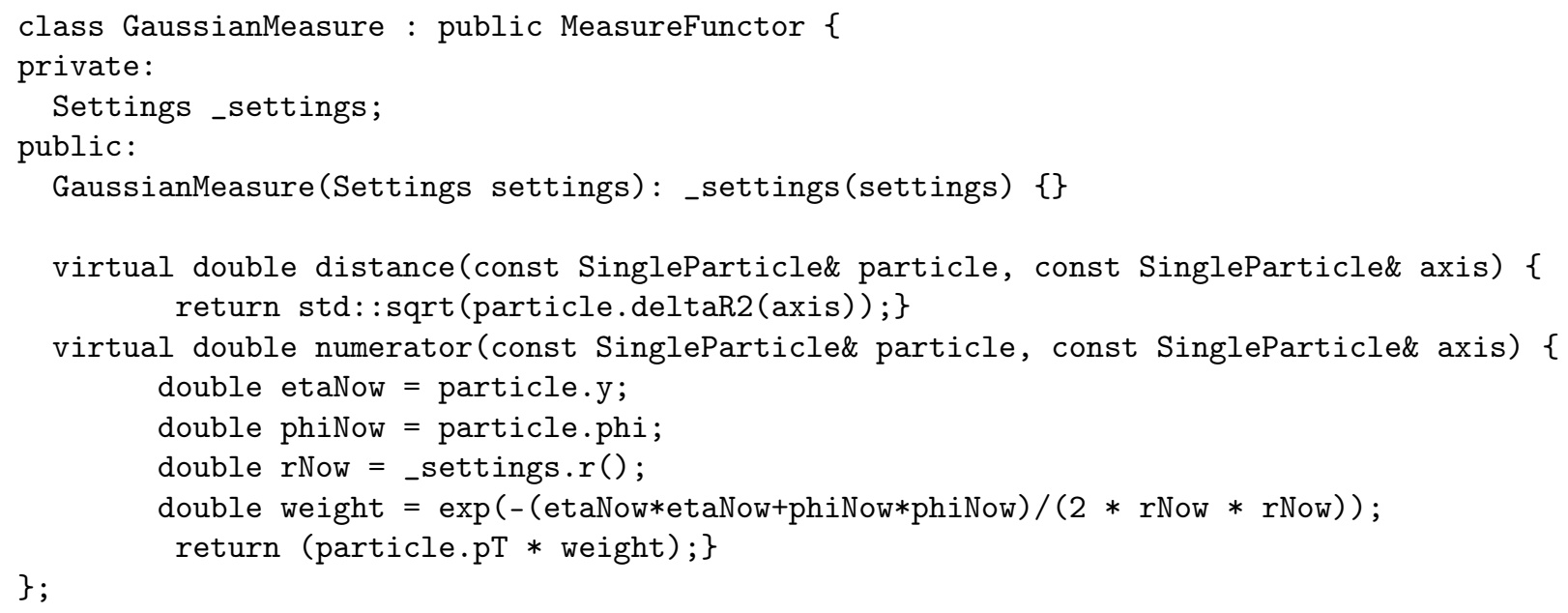




\section{C.2 Functions}

\section{C.2.1 matchTemplate}

The matchTemplate function is used to locate patterns inside the observed energy distributions within jets which have good overlap ("match") to the set of templates. The algorithm can handle a variety of complicated patterns, e.g., when the templates have more than the minimum number of partons or when there are additional kinematical constraints, such as the $W$ mass in a hadronically decaying top quark. The algorithm is implemented in the matchTemplate function whose prototype looks like this:

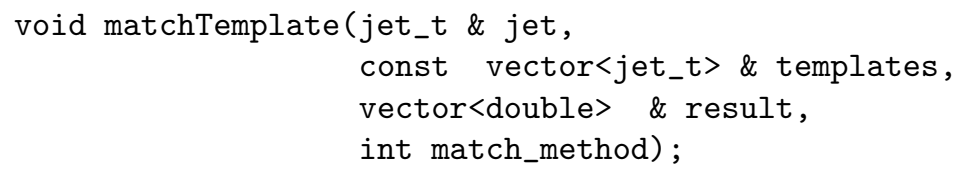

The function arguments have the following meaning:

- jet: The jet being analyzed. It must be a vector of SingleParticle.

- templates: Comparison template catalog. It must not have more particles than the source event.

- result: Map of comparison results. It must be a one-dimensional vector of double. After the search, its dimension is templates.size().

- match_method: Parameter specifying the functional measure or comparison method. It can take values available in Jet_shape_scheme. See Table 1 for details.

\section{C.2.2 maximize}

Finds the global maximum in the result array and its position.

void maximize(const vector<double> \& result, double \& maxVal, int \& maxLoc );

The parameters have the following meaning:

- result: input array

- maxVal: pointer to the returned maximum value; NULL is used if not required.

- maxLoc: pointer to the returned maximum location

\section{C.2.3 ConvertToPseudoJet}

For internal use. Converts a jet_t to PseudoJet.

std: : vector<fastjet: :PseudoJet> ConvertToPseudoJet(const jet_t\& particles); 


\section{C.2.4 ConvertToMat}

For internal use. Converts a PseudoJet to jet_t.

jet_t ConvertToMat(const fastjet::PseudoJet\& jet);

\section{C.2.5 overlapDistance}

Calculates the overlap between two jets or templates in the $\eta, \phi, p_{T}$ space, assuming one jet is a "template". RO is the template sub-cone radius.

double overlapDistance (jet_t \& jet1, jet_t \& jet2, double R0) ;

\section{C.2.6 reset}

For internal use. Clears internal flags in a jet for reuse.

void reset (jet_t \& jet);

\section{C.2.7 eShape}

In some applications, one would like to have a more realistic model for the energy profile of a template parton than simply a fixed cone. TemplateTAGger provides with a simple scaling rule for the template subcone radius that draws information from jet shape measurements at the LHC [31]. Note that the ATLAS jet shape study contains no data points for $p_{T}<30 \mathrm{GeV}$. The eShape extrapolates into the low $p_{T}$ region, with the constraint that the maximum value for the template radius is 0.3 . See Ref. [26] for more detail.

To compute the energy profile of a template parton, the numerical values for the integrated jet shape measured by ATLAS are fit in different regions of jet $p_{T}$. eShape returns the radius, $r$, that is needed to contain $80 \%$ of the transverse momentum in a cone of radius $r$ around the template parton direction.

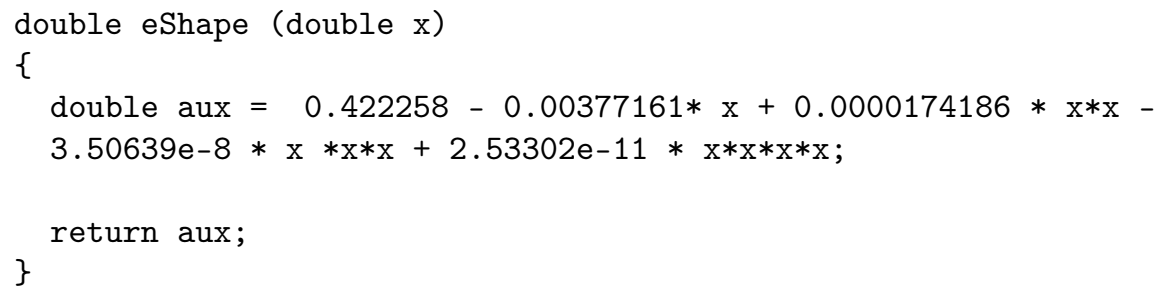




\section{References}

[1] S.D. Ellis, J. Huston, K. Hatakeyama, P. Loch, and M. Tonnesmann. Jets in hadron-hadron collisions. Prog.Part.Nucl.Phys., 60:484-551, 2008.

[2] A. Abdesselam, E. Bergeaas Kuutmann, U. Bitenc, G. Brooijmans, J. Butterworth, et al. Boosted objects: A Probe of beyond the Standard Model physics. Eur.Phys.J., C71:1661, 2011.

[3] A. Altheimer, S. Arora, L. Asquith, G. Brooijmans, J. Butterworth, et al. Jet Substructure at the Tevatron and LHC: New results, new tools, new benchmarks. J.Phys., G39:063001, 2012.

[4] Gavin P. Salam. Towards Jetography. Eur.Phys.J., C67:637-686, 2010.

[5] Pran Nath, Brent D. Nelson, Hooman Davoudiasl, Bhaskar Dutta, Daniel Feldman, et al. The Hunt for New Physics at the Large Hadron Collider. Nucl.Phys.Proc.Suppl., 200-202:185-417, 2010.

[6] Tilman Plehn and Michael Spannowsky. Top Tagging. J.Phys., G39:083001, 2012.

[7] Jesse Thaler and Ken Van Tilburg. Maximizing Boosted Top Identification by Minimizing N-subjettiness. JHEP, 1202:093, 2012.

[8] Chunhui Chen. New approach to identifying boosted hadronically-decaying particle using jet substructure in its center-of-mass frame. Phys.Rev., D85:034007, 2012.

[9] Zhenyu Han. Tracking the Identities of Boosted Particles. Phys.Rev., D86:014026, 2012.

[10] Ilya Feige, Matthew D. Schwartz, Iain W. Stewart, and Jesse Thaler. Precision Jet Substructure from Boosted Event Shapes. Phys.Rev.Lett., 109:092001, 2012.

[11] Tilman Plehn, Gavin P. Salam, and Michael Spannowsky. Fat Jets for a Light Higgs. Phys.Rev.Lett., 104:111801, 2010.

[12] Tilman Plehn, Michael Spannowsky, and Michihisa Takeuchi. How to Improve Top Tagging. Phys.Rev., D85:034029, 2012.

[13] Tilman Plehn, Michael Spannowsky, and Michihisa Takeuchi. Boosted Semileptonic Tops in Stop Decays. JHEP, 1105:135, 2011.

[14] Jesse Thaler and Ken Van Tilburg. Identifying Boosted Objects with N-subjettiness. JHEP, 1103:015, 2011.

[15] Yanou Cui, Zhenyu Han, and Matthew D. Schwartz. W-jet Tagging: Optimizing the Identification of Boosted Hadronically-Decaying W Bosons. Phys.Rev., D83:074023, 2011.

[16] Anson Hook, Martin Jankowiak, and Jay G. Wacker. Jet Dipolarity: Top Tagging with Color Flow. JHEP, 1204:007, 2012.

[17] Davison E. Soper and Michael Spannowsky. Finding top quarks with shower deconstruction. 2012. 
[18] David E. Kaplan, Keith Rehermann, Matthew D. Schwartz, and Brock Tweedie. Top Tagging: A Method for Identifying Boosted Hadronically Decaying Top Quarks. Phys.Rev.Lett., 101:142001, 2008.

[19] Jason Gallicchio and Matthew D. Schwartz. Seeing in Color: Jet Superstructure. Phys.Rev.Lett., 105:022001, 2010.

[20] Leandro G. Almeida, Ozan Erdogan, Jose Juknevich, Seung J. Lee, Gilad Perez, et al. Threeparticle templates for a boosted Higgs boson. Phys.Rev., D85:114046, 2012.

[21] Leandro G. Almeida, Raz Alon, and Michael Spannowsky. Structure of Fat Jets at the Tevatron and Beyond. Eur.Phys.J., C72:2113, 2012.

[22] Leandro G. Almeida, Seung J. Lee, Gilad Perez, Ilmo Sung, and Joseph Virzi. Top Jets at the LHC. Phys.Rev., D79:074012, 2009.

[23] Leandro G. Almeida, Seung J. Lee, Gilad Perez, George Sterman, and Ilmo Sung. Template Overlap Method for Massive Jets. Phys.Rev., D82:054034, 2010.

[24] Matteo Cacciari, Gavin P. Salam, and Gregory Soyez. The Anti-k(t) jet clustering algorithm. JHEP, 0804:063, 2008.

[25] Gregory Soyez, Gavin P. Salam, Jihun Kim, Souvik Dutta, and Matteo Cacciari. Pileup subtraction for jet shapes. 2012.

[26] Mihailo Backovic, Jose Juknevich, and Gilad Perez. Boosting the Standard Model Higgs Signal with the Template Overlap Method. 2012.

[27] Leandro G. Almeida, Ozan Erdogan, Jose Juknevich, Seung J. Lee, Gilad Perez, et al. Threeparticle templates for a boosted Higgs boson. Phys.Rev., D85:114046, 2012.

[28] Jesse Thaler and Lian-Tao Wang. Strategies to Identify Boosted Tops. JHEP, 0807:092, 2008.

[29] Leandro G. Almeida, Seung J. Lee, Gilad Perez, George F. Sterman, Ilmo Sung, et al. Substructure of high- $p_{T}$ Jets at the LHC. Phys.Rev., D79:074017, 2009.

[30] Guy Gur-Ari, Michele Papucci, and Gilad Perez. Classification of Energy Flow Observables in Narrow Jets. 2011.

[31] G. Aad et al. Study of Jet Shapes in Inclusive Jet Production in $p p$ Collisions at $\sqrt{s}=7 \mathrm{TeV}$ using the ATLAS Detector. Phys.Rev., D83:052003, 2011. 